
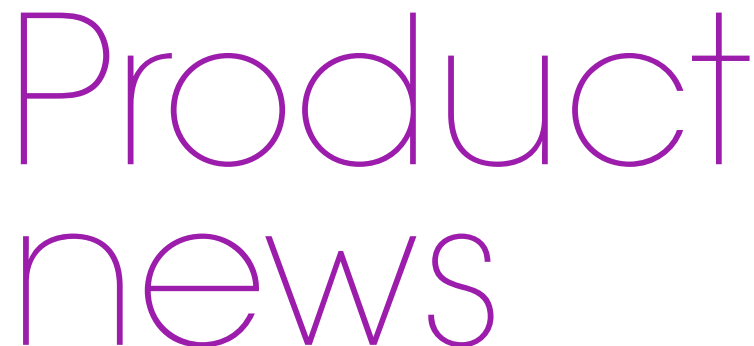

Product news is provided as a service to readers using text and images from

the manufacturer, supplier or distributor and does not imply endorsement by

BDJ Team. Normal and prudent research should be exercised before purchase

or use of any product mentioned.

\section{KEY OPINION LEADER PREVIEWS NEW SONIC BRUSH}

Anna Middleton has turned her work as a dental hygienist into a brand. She has set herself up as 'London Hygienist Ltd' and has become a multi-award winning and influential dental professional with a thriving business. She works as a Key Opinion Leader (KOL) for leading dental companies and has a flourishing social media presence.

As one of Philips KOLs, Anna was invited to visit its world HQ in Seattle, Washington. Over two days she, along with fellow hygienist Laura McClune, were given an insight into the development of the first Sonicare toothbrush 25 years ago, as a precursor to learning about the latest Sonicare 7500 ExpertClean which is about to be launched in the UK.

This new brush, with its connected technology, is designed to guide patients to better oral care habits. Sensors track and measure patients' brushing behaviours while pressure sensors in the brush handle provide instant feedback and guide the patient to adjust their brushing style. Over time, the Sonicare app retains and analyses these habits and generates a progress report for both them and their dental professional, to help develop and maintain better brushing technique. The

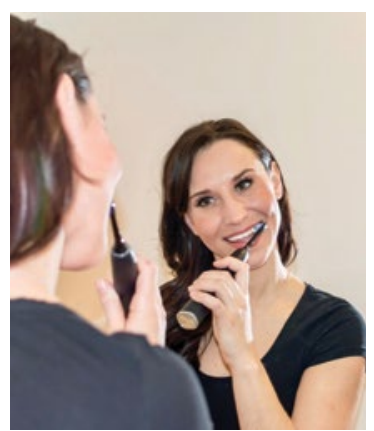

ExpertClean brush comes with a new head featuring soft, flexible bristles which are designed to curve around the contours of the teeth, giving four times more surface contact and up to ten times more plaque removal [than a manual toothbrush] from hard-toreach spots. Studies show that people who use this brush head have up to $100 \%$ less gum inflammation and up to $7 x$ healthier gums [than using a manual toothbrush] in just two weeks. Patients would have to brush for a whole month to achieve what Philips Sonicare can do in just two minutes.

For more information visit www.philips. co.uk/sonicare.
ARE YOU WORRIED ABOUT BAD REVIEWS?

We all make buying decisions based on reviews; the same goes for patients when they are choosing a dental practice.

EasyReview Pro will generate high volumes of fresh online reviews, on the platforms of your choice - you are in control!

In just ONE month, a practice using EasyReview Pro obtained:

- One hundred and thirty-five new reviews

- A 400\% increase in Google reviews

- A 30\% increase in web traffic!

EasyReview Pro set it up so you can sit back and watch your online reputation soar.

Contact Dental Design for full details of EasyReview Pro and to get your FREE trial.

To find out more about Dental Design's services, visit: https://dental-designproducts.co.uk/, email enquiries@dentaldesign.co.uk or telephone 01202677277.

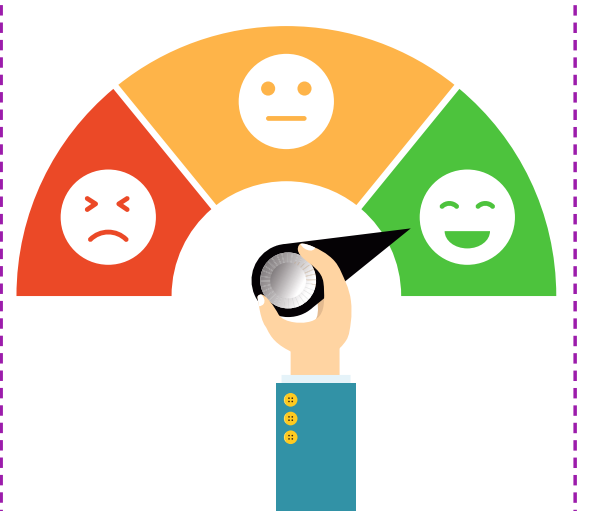

\title{
NEW DISCOUNT CARD AVAILABLE FOR DENTAL NURSES
}

Totum, powered by NUS extra, has launched a new card for members of professional associations who are studying: TOTUM PRO

Dental nurses face an increasingly demanding lifestyle, often balancing work, family and education. TOTUM PRO is a new product from OneVoice Digital, combining convenience and affordability with the best possible benefits - and is now available to British Association of Dental Nurses (BADN) Student Associate members and Full Members who are on a course of study.

The price of TOTUM PRO will be available for just $£ 19.99$ for one year, $£ 34.99$ for two years, or $£ 44.99$ for three years; BADN members will get TOTUM PRO for three months extra for free.

Discounts available to TOTUM PRO card holders include:

- $10 \%$ discount at Co-op

- $10 \%$ discount at Asos as well as other fashion retailers

- Restaurant and dinning deals, including up to $30 \%$ off at Zizzis and $20 \%$ off Pizza Hut

- $10 \%$ off at Superdrug (with your Health and Beauty card)
The existing TOTUM powered by NUS extra card will remain available only to students with an academic email address (ie @university.ac.uk). BADN members who already have a TOTUM card will continue to enjoy the benefits of their TOTUM card until its expiry date and at renewal can apply for a TOTUM PRO card. Other BADN members were able to purchase TOTUM PRO to access the exciting, relevant discounts at the special price of $£ 14.99$ for 15 months (until 16 September 2019) and can purchase it for $£ 19.99$ for 15 months thereafter. 